\title{
A mixed methods needs assessment of pediatric emergency and critical care in Tanzanian providers: a model for curriculum development
}

\author{
Carol C Chen 1 , Steven Straube1, Holly Vo², Upendo George ${ }^{3}$, Hendry Sawe ${ }^{3}$, Andrea G Tenner ${ }^{1}$ \\ 1 Department of Emergency Medicine, University of California, San Francisco, California, USA, ${ }^{2}$ Steven Straube University of Washington/Seattle \\ Children's Hospital Pediatric Pulmonary \& Sleep Medicine Division, Seattle, Washington, USA, ${ }^{3}$ Department of Emergency Medicine, Muhimbili \\ National Hospital, Dar es Salaam, Tanzania \\ Keywords: curriculum development, tanzania, critical care, pediatric emergency \\ https://doi.org/10.29392/001c.13387
}

\section{Journal of Global Health Reports}

Vol. 4, 2020

\section{Background}

There are several curricula developed to train providers in low-resource settings on how to care for critically ill and injured children, however few incorporate the unique needs, insights, and challenges faced by the providers. Using a mixed methods approach, we conducted a needs assessment to determine the most important learning issues, attitudes, and beliefs of emergency department (ED) providers at Muhimbili National Hospital (MNH) about the development of a novel pediatric emergency medicine (PEM) curriculum for their setting.

\section{Methods}

A retrospective, observational, analysis of a database of pediatric ( $<18$ years old) ED visits from April 2016 to April 2017 identified the most common presenting diagnoses.

Qualitative focus groups and semi-structured interviews were conducted using purposive sampling of providers. Content analysis identified emerging themes and member-checking was performed approximately 5 months later.

\section{Results}

Analysis of 16005 visits showed that 8 of the top 15 most common diagnoses were shared with the 15 most important topics identified by healthcare providers from the qualitative analysis; sepsis, anemia, malnutrition, congenital heart disease, pneumonia, burns, malaria, and seizures. Twenty-one healthcare providers (2 ED attending physicians, 1 pediatric attending, 5 ED residents, 9 nurses, and 4 registrars) participated and thematic saturation was achieved. Fifty-seven percent of respondents $(n=13)$ preferred a course taught in-person, and 58\% ( $\mathrm{n}=15)$ preferred a course length of less than six months. Respondents preferred a mixed instruction approach of lectures and didactics with simulation and skills stations. Three principal perspectives on poor patient outcomes were identified; i) delayed presentation to care from a lack of recognition of disease severity and/or inappropriate or inadequate treatment; ii) poor communication and a lack of knowledge of pediatric resuscitation and critical care, and limited pediatric supplies and equipment; and iii) inadequate inpatient care despite ED stabilization.

\section{Conclusions}

Emergency care providers reported interest in participating in a short PEM curriculum with both live training and self-administered learning. The topics identified were concordant between participants and a database analysis, as well as previously published studies. Themes on poor patient outcomes have provided a deeper context into which the curriculum will be taught. These findings can inform future curriculum development efforts.

Emergency care is defined by the World Health Organization (WHO) as care for the acutely ill and injured delivered by frontline providers who manage medical, surgical, and obstetric emergencies, relying on early recognition and resuscitation. ${ }^{1}$ A 2006 WHO report examining the barriers to adequate emergency care found that the lack of trained medical professionals was a significant barrier. ${ }^{2}$ Subsequently, the World Health Assembly passed a resolution calling for the improvement of emergency care access and availability across the globe. ${ }^{3,4}$ 
Since its recognition there has been increasing efforts to develop and implement formal emergency care training programs however such systems are still in their infancy in many countries in sub-Saharan Africa. 5,6

It is estimated that up to $27 \%$ of all emergency visits involve patients less than 13 years old, yet there are still no dedicated, formal Pediatric Emergency Medicine (PEM) training programs in sub-Saharan Africa. ${ }^{7-9}$ This number is likely to increase as the population of the region is expected to continue trending towards a younger subset. ${ }^{10}$ Timely and efficient emergency care has been identified as an important factor in improving survival rates in critically ill and injured children. ${ }^{11}$ There have been previously published proposals for pediatric emergency care training, however no studies to date have dived deeper to investigate the unique needs and challenges faced by frontline providers in subSaharan Africa, and how these shape their practice. 6,12 While increasing access to pediatric-specific training is important, it is imperative to understand the context into which the training must be delivered in order to ensure that it is relevant and effective for target providers working in low- and middle-income countries. ${ }^{13}$

In 2017, the African Federation for Emergency Medicine (AFEM), a professional organization with over 2000 members dedicated to bringing high-quality emergency care to the continent, convened a working group to develop an open-access, pediatric-specific emergency care curriculum. A well-established protocol was used to guide the curriculum development process, for which one of the first steps was a needs assessment to understand the most important needs of target learners. ${ }^{14}$

We employed a mixed methods approach to conduct a needs assessment study to determine the most important learning issues, attitudes, and beliefs of five different groups of emergency department (ED) providers at a large, public referral hospital in Dar es Salaam, Tanzania. Specifically, we sought to identify the most common pediatric emergencies, describe perceived factors affecting poor pediatric patient outcomes, and understand specific training needs, such as preferred learning formats and modalities. To obtain the most common pediatric emergencies, we compared the most common pediatric emergencies reported by providers with the final diagnoses of all pediatric visits to the emergency department over a 1-year period. All of this information would be used to shape the learning objectives and overall development of the curriculum.

\section{METHODS}

\section{STUDY DESIGN}

To develop the learning objectives of the curriculum, we needed to understand the most common pediatric cases presenting to the emergency department, as well as the pediatric emergencies associated with the most morbidity and mortality. We sought to obtain this information in two ways: by asking providers themselves during qualitative interviews and focus groups (every time a participant mentioned a diagnosis, it was tallied), and by analyzing all pediatric visits to the emergency department over one year. Thus, this mixed methods study consisted of qualitative se- mi-structured interviews and focus groups conducted with a convenience sample of emergency care providers, augmented by quantitative data obtained from a retrospective, observational database analysis of all pediatric ED visits ( 0 to 17 years of age) during a 12-month period.

Information on providers' preferred learning format and their attitudes and belief about factors affecting poor pediatric outcomes was also obtained during the qualitative interviews and focus groups.

The study protocol and consent procedures were approved by the Institutional Review Boards of Muhimbili National Hospital and the University of California at San Francisco. All participants provided informed consent verbally with interpreters available if needed.

\section{DATABASE ANALYSIS}

A cross-sectional, retrospective analysis of a database of pediatric ( $<18$ years old) visits for one year, from April 2016 to April 2017, was conducted using the electronic medical record system of the hospital's ED. We sought to capture annualized data without a pre-specified number of visits.

Patient visits were grouped by the providers' diagnoses into fifteen categories based on system involvement and then ranked according to incidence. Secondary sorting grouped the most common diagnoses by system involved. The diagnoses were then compared with the qualitative data to assess for accuracy.

All ED visits for patients between the ages of 0 and 18 years were included. Data was de-identified and stored on secure digital platforms.

\section{PARTICIPANTS}

Recognizing that much of emergency care provided in Tanzania, as in many low-resource settings, is provided by health care workers not specifically trained in emergency care, we included a purposive sample of members of several different provider groups. ${ }^{15}$ These groups were developed a priori after discussions with local leaders and repeated observation of care pathways, and were determined to be representative of the primary workers providing emergency care in this setting. Specifically, study participants were members of the groups: (i) emergency department nurses; (ii) emergency department registrars; (iii) resident physicians; (iv) pediatric attending physicians; (v) emergency department attending physicians. These groups were identified as those who would benefit most from a novel pediatric emergency medicine curriculum.

Twenty-one emergency care providers were recruited from 5 different provider groups described above to participate in the interviews and focus groups. Emergency department registrars are practicing physicians who have completed an intern year. All were 18 years or older. Researchers identified potential participants using the snowball technique, whereby one stakeholder group member subsequently identified another group member to participate in the study. ${ }^{16}$ 
Table 1. Pediatric visits to an emergency department over 1 year by: A) Most commonly affected organ system, and B) Fifteen most common discharge diagnoses

\begin{tabular}{|c|c|c|c|}
\hline \multicolumn{2}{|c|}{ A } & \multicolumn{2}{|c|}{ B } \\
\hline Organ system & Number of visits (\%) & Discharge diagnosis & Number of visits (\%) \\
\hline Infectious Disease & $6,777(42.9)$ & Malaria & $1,644(10.4)$ \\
\hline Hematology/Oncology & $1,268(8.0)$ & Pneumonia & $1,239(7.8)$ \\
\hline Gastroenterology & $1,189(7.5)$ & Fractures & $985(6.2)$ \\
\hline Orthopedics & $1,095(6.9)$ & Upper Respiratory Infections & $926(5.9)$ \\
\hline Cardiology & $1,084(6.9)$ & Congenital Heart Disease & $876(5.5)$ \\
\hline Neurology & $1,005(6.4)$ & Sepsis & $797(5.0)$ \\
\hline Otolaryngology (ENT) & $828(5.2)$ & Urinary Tract Infection & $682(4.3)$ \\
\hline Urology/Nephrology & $410(2.6)$ & Anemia and Sickle Cell & $639(4.0)$ \\
\hline Ophthalmology & $245(1.6)$ & Diarrhea and Gastroenteritis & $443(2.8)$ \\
\hline Pulmonology & $166(1.1)$ & Burns & $435(2.8)$ \\
\hline Rheumatology & $126(0.8)$ & Seizures & $432(2.7)$ \\
\hline Endocrinology & $99(0.6)$ & Malnutrition & $426(2.7)$ \\
\hline Dermatology & $96(0.6)$ & Foreign Bodies & $350(2.2)$ \\
\hline Immunology & $85(0.5)$ & Hernia & $296(1.9)$ \\
\hline Other & $1,317(8.3)$ & Dehydration & $274(1.7)$ \\
\hline Total & $15,790(100)$ & Total & $10,444(66.1)$ \\
\hline
\end{tabular}

\section{INTERVIEWS, FOCUS GROUPS, AND QUESTIONNAIRES}

A guide for all focus groups and interviews was developed, pilot-tested and revised during several research team meetings. The same interviewer (CCC) with previous experience conducted all interviews and focus groups.

The intrinsic nature of each provider group dictated the method by which data was collected and provided "triangulation." 17 ED resident physicians and ED nurses represented a large and diverse constituency; therefore, focus groups with these constituents were deemed ideal for the collection of data. Due to small numbers of available participants in other groups, interviews were conducted with these groups instead of focus groups.

Questions focused on provider preferences for learning modalities and course format, the most common perceived pediatric diagnoses, and factors affecting poor pediatric patient outcomes.

After permission was obtained from participants, notes from all focus groups and interviews were coded by investigators and staff trained in issues of confidentiality. These sessions were conducted in a private hospital setting in English; interpreter services were available but not required.

\section{INTERVIEW/FOCUS GROUP ANALYSIS}

Researchers used "constant comparison" analysis, whereby they repeatedly read through notes, formulated an initial framework of key codes, and refined these codes with successive readings. ${ }^{17,18}$ They developed mutually agreed-upon definitions and examples for each code. Codes were reviewed and revised with disagreements in coding resolved by team consensus. Memos of coding decisions were kept to provide consistency in coding as analysis progressed.

The "thematic approach", a widely utilized process in the analysis of qualitative data, was used to identify themes of factors contributing to poor pediatric patient outcomes. ${ }^{16,17}$ Content "saturation" was achieved when no new themes emerged in successive focus group or interview transcripts. ${ }^{19}$

"Member checking" was performed approximately 5 months after the initial data collection period to check for accuracy and completeness of the findings; no new themes were discovered. ${ }^{20}$

\section{RESULTS}

\section{DATABASE ANALYSIS}

A total of 16,005 pediatric visits were identified during a one-year period from April 2016 to April 2017. These visits were grouped into fifteen subcategories based upon organ system involved and sorted from most frequent to least frequent. Further, the visits were also categorized and sorted by the discharge diagnosis from most common to least common (Table 1).

\section{INTERVIEWS AND FOCUS GROUPS}

A total of twenty-one Muhimbili National Hospital (MNH) ED providers (2 ED attendings, 1 pediatric attending, 5 ED residents, 9 ED nurses, and 4 ED registrars) participated in the focus groups and semi-structured interviews.

Preferred course length was less than 6 months $(n=15$, $57.8 \%)$ as opposed to $6-12$ months $(n=7,27 \%)$ or more than 12 months $(\mathrm{n}=4,15.4 \%)$. The preferred course format was 
Table 2. Comparison of reported most common and important pediatric emergency diagnoses presenting to the MNH ED (Qualitative - interviews and focus groups) with historical presenting diagnoses (Quantitative - database analysis)

\begin{tabular}{|c|c|c|c|}
\hline \multicolumn{2}{|c|}{ Qualitative (interviews and focus groups) } & \multicolumn{2}{|c|}{ Quantitative (database analysis) } \\
\hline Topics & Total & Diagnosis & Number \\
\hline Electrolyte Imbalances & 19 & Malaria & 1644 \\
\hline Sepsis & 15 & Pneumonia & 1239 \\
\hline Trauma & 15 & Fracture & 985 \\
\hline Respiratory distress & 13 & Respiratory tract infections & 926 \\
\hline Anemia & 12 & Congenital heart disease & 876 \\
\hline Malnutrition & 12 & Sepsis & 797 \\
\hline Congenital heart disease & 11 & UTI, Cystitis & 682 \\
\hline Pneumonia & 11 & Sickle cell disease & 639 \\
\hline Burns & 10 & Diarrhea, gastroenteritis & 443 \\
\hline Malaria & 9 & Burns & 435 \\
\hline Advanced Life Support skills & 8 & Seizures, Epilepsy & 432 \\
\hline Basic Life Support skills (including choking) & 8 & Malnutrition, SAM, KWA & 426 \\
\hline Diabetic Ketoacidosis & 8 & Anemia & 414 \\
\hline Procedures (PIV, intubation, IO) & 8 & Foreign bodies & 350 \\
\hline Seizure disorders & 8 & Hernia & 296 \\
\hline
\end{tabular}

IO - Intraosseous, KWA - Kwashiorkor, PIV - Peripheral IV, SAM - Severe Acute Malnutrition, UTI - Urinary Tract Infection

a live course $(\mathrm{n}=13,50 \%)$ compared with a mixed live and on-line course $(n=8,30.8 \%)$ or an on-line only course $(n=2$, $7.7 \%$ ). Of note, 26 responses were collected for these questions as five participants provided more than one response.

Participants' responses for the most common presenting pediatric emergency diagnoses were counted then ranked from most common to least common. This list represents the most common pediatric emergency diagnoses reported via our qualitative methods (interviews and focus groups). This list was compared to our list of the most common pediatric emergency diagnoses obtained from quantitative methods, or the database analysis (Table 1, Section B). Table 2 compares the lists and demonstrates that 8 (53.3\%) of the top 15 diagnoses were shared in common between the qualitative and quantitative lists, as highlighted in grey.

Three general themes emerged around factors affecting poor pediatric patient outcomes: i) delayed presentation to care; ii) care issues in the ED; and iii) Iinadequate inpatient care following ED stabilization (Figure 1).

\section{INTERVIEW/FOCUS GROUP THEMES}

\section{DELAYED PRESENTATION TO CARE}

Several participants from different provider groups cited a lack of recognition of severity of illness as one of the primary reasons for poor pediatric outcomes in their emergency department. Regarding a child who was brought in with severe kidney injury, one emergency medicine specialist stated: "Mom just doesn't notice that the child has not urinated in a while." A registrar commented about another patient, "...(s)he was very sick because have been delayed in care," then went on to highlight the importance of health literacy in families by stating, “... health-seeking behavior starts with families.” Another registrar talked about the lack of pediatric knowledge in the prehospital setting: "... [they] do not transfer until the patient is very sick."

\section{CARE ISSUES IN THE ED}

Challenges in delivering patient care in the emergency department were also brought up frequently. A common code was the lack of pediatric-specific supplies and equipment, including the rarity of pediatric ventilators. One registrar also expressed frustration over delayed treatment results: "... blood gas machine was not working so could not monitor electrolytes."

\section{INADEQUATE INPATIENT CARE FOLLOWING ED STABILIZATION}

Finally, many providers expressed frustration about the lack of "continuity of care" when a sick child was admitted to the pediatric ward. One nurse stated: "[We] need pediatric observation unit because kids deteriorate once they go up to the wards." One of the specialists pointed out a deficit in emergency care training amongst the ward staff saying, "People working on the ward do not know how to resuscitate." A lack of space on the pediatric ward was also mentioned, in addition to the recurring code of a lack of pediatric-specific equipment and supplies.

\section{DISCUSSION}

Developing and implementing effective pediatric emer- 
THEMES

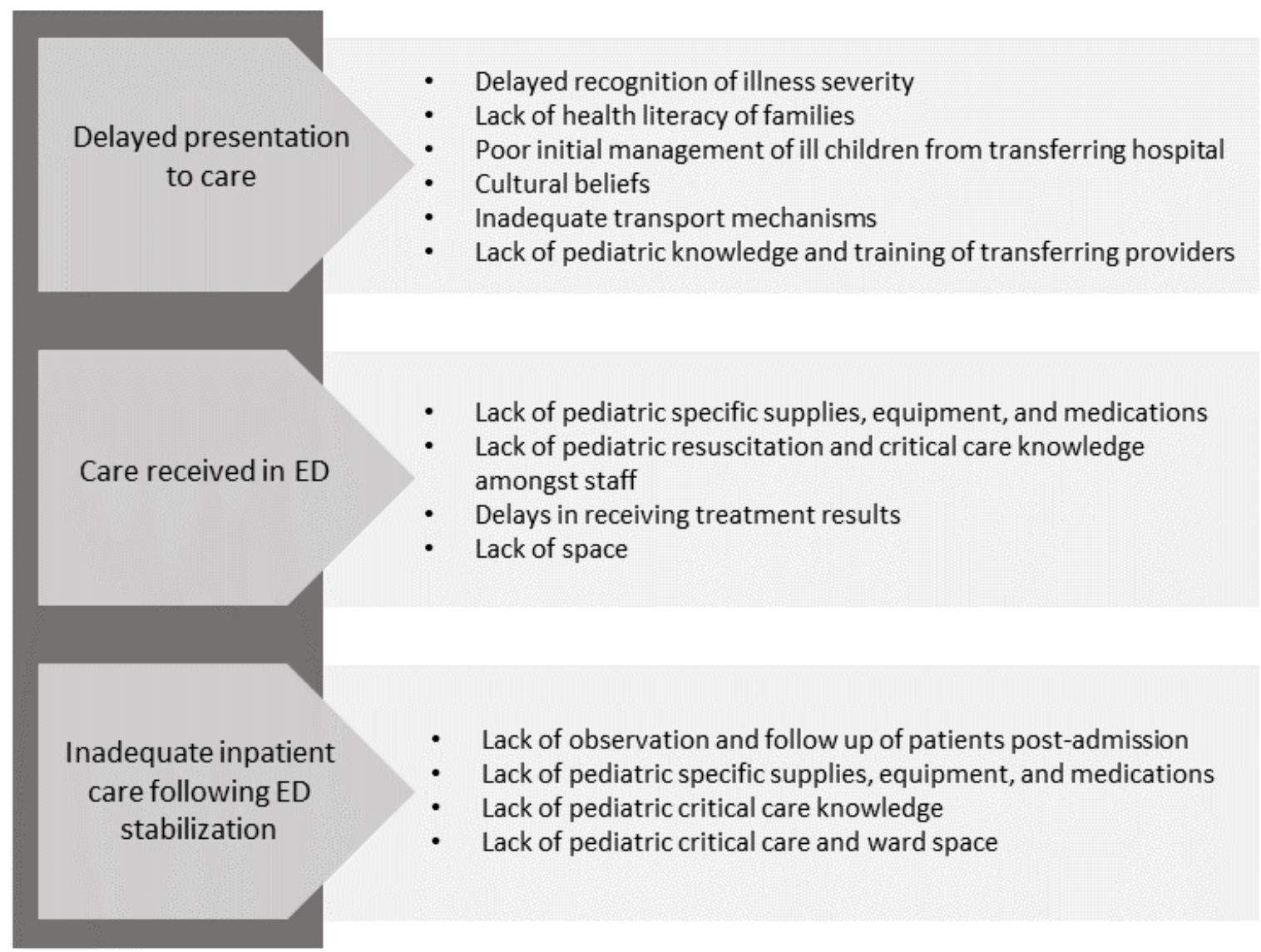

\section{CODES}

- Delayed recognition of illness severity

Lack of health literacy of families

g hospital

tural beliefs

- Lack of pediatric specific supplies, equipment, and medications amongst staff

Delays in receiving treatment results

Lack of space

Lack of observation and follow up of patients post-admission

Lack of pediatric specific supplies, equipment, and medications

Lack of pediatric critical care knowledge

Lack of pediatric critical care and ward space

Figure 1. Main perceived factors affecting poor pediatric outcomes among critically ill and injured patients in the ED.

gency care is imperative to improve healthcare outcomes in low- and middle-income countries. ${ }^{11}$ It is also necessary to tailor educational programs to address specific needs and issues faced by the frontline providers. ${ }^{13}$

Data help to inform the key educational topics for such providers. Several studies have used needs assessments to identify the most common pediatric emergencies in low-resource settings, and our study shows an alignment of topics including pneumonia and other respiratory illnesses, sepsis, heart disease, malnutrition, gastroenteritis, malaria, fractures, anemia, and seizure disorders. ${ }^{21-25}$ Additionally, our quantitative data demonstrate that a majority of perceived key pediatric topics are reflective of actual patient presentations.

Our data indicate that poor pediatric health outcomes are multifactorial but a recurring theme is inadequately trained providers, which highlights the importance of effective curricula. In addition, providers from different cadres identified poor training across all three settings of care (pre-hospital setting, emergency department, and wards) as a significant factor affecting patient outcomes.

While special considerations for managing pediatric emergencies in such settings has been previously listed, our needs assessment goes further to try to understand these unique challenges so that our curriculum can attempt to accommodate them and thereby become more relevant to our target audience. ${ }^{25}$ For example, as mentioned, a lack of pediatric-specific knowledge was identified in both the pre-hospital and ED setting. Many providers interviewed expressed frustration with receiving critically ill patients whose care had been delayed due to a lack of recognition of illness severity. In response to this, the working group expanded its mission to develop a curriculum for more than just physicians. A curriculum for 3 different tiers of emergency care providers is being developed, with the first tier to include prehospital providers.

In addition, our needs assessment examined preferred learning modalities and formats as well as content, which has not been previously described. The majority of emergency care providers in our target audience are working professionals who may not have the time or resources to take part in a lengthy, in-person, formal training program. Our study confirmed this supposition by showing that more than half of the participants preferred a shorter training program, or one that lasted less than 6 months. Participants also favored either live, in-person training or a combination of live and on-line education. Only $7.7 \%$ preferred an online only format. Therefore, a mixed, asynchronous curriculum design was chosen whereby short (less than 30 minutes), narrated lectures available on-line would be complemented by in-person skills stations and simulated scenarios. 
We believe the findings in our study can be used to improve pediatric emergency care in Tanzania by first, tailoring an educational course to the needs of providers including a short ( $<6$ month) live training course. Secondly, this course should address the perceived and actual most common presenting conditions, making it relevant to the everyday care of critically ill and injured children in this setting. Lastly, and maybe most importantly, the qualitative nature of our results elucidate perceived barriers to improved pediatric emergency care which include delays to presentation, inadequate supplies in the emergency department, and inadequate training and care among the inpatient wards. As described above, these unique insights have already provided the evidence needed to make major decisions on curriculum design and implementation. Preliminary results from the pilot study of the implementation of the curriculum for the first tier of providers in two different settings in sub-Saharan Africa has already demonstrated significant improvements in provider self-efficacy and knowledge in pediatric emergency care. ${ }^{26,27}$ Ongoing longitudinal studies are attempting to show significant differences in provider behavior and patient outcomes.

\section{SPECIFIC CHALLENGES}

We encountered several challenges in conducting this needs assessment that may be of instructive value. First, recruiting participants was difficult given the apprehension of many providers, especially those lower on the scale of perceived power dynamic, such as nurses and registrars. These individuals were also more likely to experience a language barrier, which also made them more hesitant to participate, despite having an interpreter present. For this reason, we used the snowball technique to recruit participants, allowing them to identify additional participants. The limitations of this technique are discussed below, however it has been considered very effective by other authors given the history of mistrust of Western organizations that exists in many similar settings. ${ }^{28}$ It was also difficult to recruit attending physicians as participants, mainly because there are so few of them, with only one emergency department attending working at any given time, and only one pediatric attending physician in the entire hospital. Secondly, avoiding response bias was a challenge as many participants were eager to provide responses that were viewed as more desirable. To address this, we attempted to build trust by choosing primary interviewers with repeated experience in Tanzania and established relationships with the institution, and trained them in qualitative interviewing techniques. Further, responses during focus groups were subject to "groupthink" in which participants may have the tendency to agree with other participants' responses potentially suppressing individual or unique responses. We attempted to overcome this by recruiting a diverse groups of peer participants for each focus group combined with impartial and trained interviewers as described above. Lastly, retrospectively analyzing the dataset from over 16,000 emergency department visits for final diagnoses was complicated. While the existence of an electronic medical system, a rarity in many low-resource settings, made obtaining the data much easier, many of the final diagnoses had been free- texted, rather than chosen based upon a pre-determined set of variables. Therefore, each diagnosis had to be interpreted and categorized individually by researchers, which was both time- and resource-intensive.

\section{LIMITATIONS}

Our study uses a mixed methods approach integrating both qualitative and quantitative data. Our quantitative data provided in the database analysis is limited due to its retrospective nature, its origin in a single site, and the limits presented by analyzing electronic health records data, namely that there was no diagnostic hierarchy to determine the primary presenting diagnosis and that the diagnoses were written in free text, thus misspelled and/or abbreviated diagnoses could have been missed. However, MNH is the tertiary referral center for Dar es Salaam and likely represents the majority of pediatric emergency presentations for the capital city. For our qualitative data, several limitations are already discussed above, including sampling and response bias. We also attempted to overcome these biases by conducting interviews until saturation was achieved, followed by member checking. Interviews were conducted in English, which is a second language for most providers, and may have affected results. Lastly, our study only included one large, academic, referral hospital with an emergency medicine residency, and did not incorporate input from regional or district providers, which may limit the generalizability of our results.

\section{CONCLUSIONS}

Despite its limitations, our needs assessment indicates that pediatric emergency visits in a large public hospital in Tanzania span a broad range of diagnoses which correlate with the most common conditions seen in previous publications. Any curriculum developed to improve the care of critically ill or injured children in low-resource settings needs to take into account not only these conditions, but also the factors associated with poor pediatric patient outcomes, which provide a context into which the curriculum should be taught. Our results provide a window into these factors, in addition to showing that providers prefer a short, in-person, skills-focused course. These lessons can be used to guide future curriculum development efforts in similar settings.

Acknowledgements: The authors would like to thank the faculty and staff in the Emergency Department at Muhimbili National Hospital for sharing your thoughts and feelings with us. The authors of this manuscript do not have any sources of bias in terms of affiliations, funding sources, or financial/management relationships.

Ethics: The Institutional Review Boards of the University of California at San Francisco and Muhimbili National Hospital reviewed and approved the study and its methods. 
Funding: There were no external sources of support for this study.

Authorship contributions: All listed authors have contributed substantially to the design, data gathering, analysis, and/or interpretation of the study. All listed authors have reviewed, edited, and approved the submitted manuscript.

Competing interests: The authors have completed the Unified Competing Interest form and have no conflicts of interest to declare.

\section{Correspondence to:}

Carol C. Chen, MD MPH

Assistant Clinical Professor of Pediatrics and Emergency Medicine

Department of Emergency Medicine Global Health Sciences Affiliate Faculty

University of California, San Francisco

carol.chen@ucsf.edu

Phone: (415) 476-3356

This is an open-access article distributed under the terms of the Creative Commons Attribution 4.0 International License (CCBY-4.0). View this license's legal deed at http://creativecommons.org/licenses/by/4.0 and legal code at http://creativecommons.org/licenses/by/4.0/legalcode for more information. 


\section{REFERENCES}

1. World Health Organization. Emergency Care. World Health Organization. https://www.who.int/emergenc ycare/en/. Published July 18, 2019.

2. World Health Organization. Sixtieth World Health Assembly. Resolution WHA 60.22: Emergency-Care Systems. Geneva: World Health Organization; 2007.

3. Bongaarts J. World Health Organization: Working Together for Health: The World Health Report 2006. Population and Development Review.

2006;32(4):790-792.

4. Anderson PD et al. World Health Assembly Resolution 60.22 and its importance as a health care policy tool for improving emergency care access and availability globally. Annals of emergency medicine. 2012;60(1):35-44. doi:10.1016/i.annemergmed.2011.1 $\underline{0.018}$

5. World Health Organization. Piloting the WHO Basic Emergency Care course in Uganda, United Republic of Tanzania and Zambia. World Health Organization. December 2015. https://www.who.int/e mergencycare/dispatches/pilot-bec-course/en/.

6. Fant CD, Schwartz KR, Patel H, et al. developing and implementing a Pediatric emergency Care Curriculum for Providers at district level Hospitals in sub-saharan africa: A Case study in Kenya. Front Public Health. 2017;5. doi:10.3389/fpubh.2017.00322

7. Walker DM, Tolentino VR, Teach SJ. Trends and challenges in international pediatric emergency medicine. Current opinion in pediatrics. 2007;19(3):247-252. doi:10.1097/MOP.0b013e3281290 $\underline{8 \mathrm{~b} 8}$

8. Wallis LA, Twomey M. Workload and casemix in Cape Town emergency departments. South African Medical Journal. 2007;97(12):1276-1280.

9. Wachira BW, Wallis LA, Geduld H. An analysis of the clinical practice of emergency medicine in public emergency departments in Kenya. Emerg Med J. 2012;29(6):473-476. doi:10.1136/emi.2011.113753

10. Ighobor K. Africa's youth: Ticking time bomb or an opportunity? Africa Renewal. 2013;27(1):10-12. do i:10.18356/be3157d6-en

11. Razzak JA, Kellermann AL. Emergency medical care in developing countries: Is it worthwhile? Bulletin of the World Health Organization. 2002;80:900-905.
12. 2012 International Standards of Care for Children in Emergency Departments. International Federation For Emergency Medicine. June 2014. https://www.ife m.cc/wp-content/uploads/2016/03/International-Stan dards-for-Children-in-Emergency-Departments-V 2.0-June-2014-1.pdf.

13. Gaspard J, Yang C-M. Training needs assessment of health care professionals in a developing country: The example of Saint Lucia. BMC Med Educ. 2016;16(1). doi:10.1186/s12909-016-0638-9

14. Thomas PA et al., ed. Curriculum Development for Medical Education: A Six-Step Approach. JHU Press; 2016.

15. Nicks BA et al. The state of emergency medicine in the United Republic of Tanzania. African Journal of Emergency Medicine. 2012;2(3):97-102. doi:10.1016/ j.afjem.2012.06.002

16. Shaghaghi A, Bhopal RS, Sheikh A. Approaches to recruiting 'hard-to-reach' populations into research: A review of the literature. Health Promot Perspect. 2011;1:86-94.

17. Ezzy D. Qualitative Analysis: Practice and Innovation. London: Routledge; 2002.

18. Braun V, Clarke V. Using thematic analysis in psychology. Qualitative research in psychology. 2006;3(2):77-101. doi:10.1191/1478088706qp063oa

19. Denzin N, Lincoln Y. The Sage Handbook of Qualitative Research. Third Edition. Thousand Oaks, CA: Sage; 2000.

20. Cho J, Trent A. Validity in qualitative research revisited. Qualitative research. 2006;6(3):319-340. do $\mathrm{i}: 10.1177 / 1468794106065006$

21. Muluneh D, Shimelis D, Benti D. Analysis of admissions to the pediatric emergency ward of Tikur Anbessa Hospital in Addis Ababa, Ethiopia. Ethiopian Journal of Health Development. 2007;21(1). doi:10.43 14/ejhd.v21i1.10031

22. Fant CD, Schwartz KR, Patel H, et al. Developing and implementing a Pediatric emergency Care Curriculum for Providers at district level Hospitals in sub-saharan africa: A Case study in Kenya. Front Public Health. 2017;5. doi:10.3389/fpubh.2017.00322

23. Jamal SM et al. Pediatric Acute Care Training Curriculum for Resource-Limited Settings: Results of an Online Survey. Pediatric Academic Society Global Pediatric Research. 2015. 
24. Shilkofski N, Crichlow A, Rice J, et al. A standardized needs assessment tool to inform the curriculum development process for pediatric resuscitation simulation-based education in resource-limited settings. Front Pediatr. 2018;6. doi:1 0.3389/fped.2018.00037

25. Duke T, Cheema B. Paediatric emergency and acute care in resource poor settings. J Paediatr Child Health. 2016;52(2):221-226. doi:10.1111/jpc.13105

26. Chen CC, Werne AL, Osborn KA, et al. Effectiveness of a Pediatric Emergency Medicine Curriculum in a Public Tanzanian Referral Hospital. West J Emerg Med. 2019;21(1):134-140. doi:10.5811/ westjem.2019.10.44534
27. Werne A, Schmid A, Wisner JK, et al. Effectiveness of a Pediatric Emergency Medicine Curriculum at a Public Referral Hospital in Liberia, a Follow-up Comparison. Accepted at Pediatric Academic Societies Meeting. Philadelphia, PA; 2020.

28. Chen CC, Cruz AT, Honoré Goltz H, Sampayo EM. A proposed framework for sustainable international partnerships: Lessons learned in rural Uganda. J Glob Health Rep. 2019;3. doi:10.29392/joghr.3.e2019054 